\title{
The Application of Combined Camera in Low Altitude Remote Sensing With Unmanned Airship
}

\author{
LIU Mingjun \\ School of Remote Sensing and Information Engineering, \\ Wuhan University, Wuhan,China \\ lmj109@yahoo.com.cn
}

\author{
SU Guozhong, LIN Zongjian, \\ Chinese Academy of Surveying and Mapping, \\ Bejing, China \\ 174083588@qq.com, lincasm@casm.ac.cn
}

\begin{abstract}
This paper describes the low altitude unmanned airship remote sensing technology, with the wide angle combined camera, high resolution and wide images are obtained. By adopting combined camera self-calibration technology, the distortion and error of wide virtual combined image are eliminated or constricted to be negligible. The project of large scale topographic mapping was performed by using the high resolution and wide images, 1:500 scale map, DEM and DOM are produced, and the result accuracy meets the national standard requirement. This technology solves the problem that the elevation accuracy is difficult to meet the required accuracy in large scale topographic mapping by photogrammetric method, and it greatly improves the production efficiency. The experiment demonstrated that the low altitude unmanned airship remote sensing technology is a new and practical method to obtain high precision geographic information.
\end{abstract}

Index Terms-Low Altitude remote sensing, Unmanned Airship, combined camera, self-calibration.

\section{INTRODUCTION}

With the development of space technologies, aviation technologies, and sensor technologies, it is possible to obtain high resolution images, which can help us extract high precision geographic information. The satellite images such as QuickBird and GeoEye image, their resolution is $0.61 \mathrm{~m}$ and $0.41 \mathrm{~m}$ respectively. The high resolution aerial image can also be obtained by aerial camera such as DMC, UCD, and SWDC etc., whose resolution may even to be $0.1 \mathrm{~m}$ according to the flight height. But the satellite and large aeroplane are not flexible enough to obtain image and get information for disaster monitoring and assessment, and emergency management. They couldn't rapidly react in some emergency conditions. By contrast, the method of using low altitude unmanned aerial vehicle(UAV) remote sensing technology can help us quickly acquire the high resolution images and information about the area we need. Being flexible and low cost, the low altitude UAV remote sensing technology is developing very fast in recent years. In this paper, we describe the application on acquisition of high precision geographic information with low altitude Unmanned Airship integrated with wide angle combined camera. By using the low altitude remote sensing technology, we obtained the high resolution images, whose GSD(Ground Sample Distance) is $3.5 \mathrm{~cm}$, and produced 1:500 scale topographical maps, DEM sand DOMs of experimental area, and the result accuracy meets the required accuracy of national standard.

\section{LOW ALTITUDE UNMANNED AIRSHIP PLATFORM}

Using aerial remote sensing technology to obtain high precision geographic information, especially, the large scale topographic map, DEM and DOM, high resolution image is the first requirement. In recent years, the fast development of Unmanned Aerial Vehicles including UAV-Aircraft, UAV-Helicopter and Unmanned Airship provide ideal aerial platforms for low attitude aerial remote sensing. Low attitude platform could help improve the image resolution without reducing the camera pixel size, also bring additional benefits, namely, performing aerophotograph under cloud, which reduces the influence of cloud and mist. Due to the short distance between camera and the ground, this method could obtain more light flux and thus obtain high sharpness images even in cloudy weather.

To obtain high resolution images, considering the requirement of flight height and speed, unmanned airship is an ideal aerial platform. The flying principle of airships is 
aerostatics, which relies on the helium gas buoyancy for lift-off and keeping balance. Therefore, airship has a series of advantages: (1) It has the function of vertical take-off and landing, and it is not necessary to have an airport support; (2) It is flexible, easy to carry and easy to transfer from one place to another; (3) It has good safety performance, unlike fixed wing aircrafts, it has no limitation of the lowest flight speed and it could flight in low speed without any safety problems; (4)Its flying height range is wide, and it could perform safe flight and cruise between $100 \mathrm{~m}$ and $1000 \mathrm{~m}$ height; (5) It is easy to operate, even non-professional operators could operate it after a short period of training, so its flight costs and maintenance costs are low.

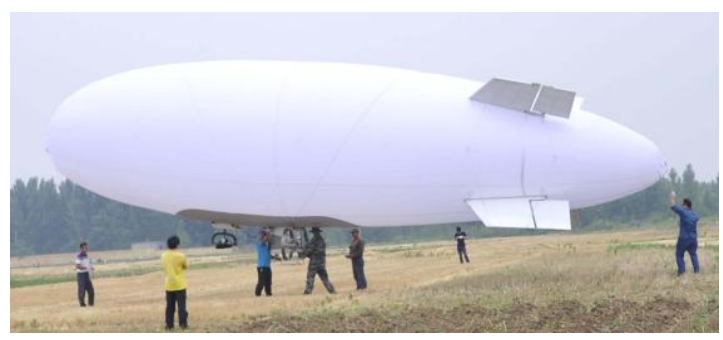

Fig.1. The Unmanned airship CK-FT180

\section{COMBINED WIDE-ANGLE CAMERA LAC-04}

Wide image is useful to improve the mapping efficiency. Large field angle of camera leads to large base-height ratio in photogrammetric mapping, which is beneficial to improve elevation measurement accuracy. Thus it is useful to enlarge the field angle of aerial camera and to increase the image size in topographical mapping. In this paper, wide image and large field angle of camera was achieved by using the method of camera combination.

\section{A. Geometric structure design of LAC-04}

The wide angle combined camera LAC-04 used in this paper is combined with four ordinary non-metric cameras of Canon 5D Mark II. Its special geometric construction can be used to self-calibration processing, which could eliminate the distortion of the combined image. The appearance and the combination principle of the camera are illustrated in the Figure 2.

$$
\left\{\begin{array}{l}
\Delta x=\left(x-x_{0}\right)\left(r^{2} k_{1}+r^{4} k_{2}+r^{6} k_{3}\right)+\left(r^{2}+2\left(x-x_{0}\right)^{2}\right) p_{1}+2\left(x-x_{0}\right)\left(y-y_{0}\right) p_{2}+\left(x-x_{0}\right) b_{1}+\left(y-y_{0}\right) b_{2} \\
\Delta y=\left(y-y_{0}\right)\left(r^{2} k_{1}+r^{4} k_{2}+r^{6} k_{3}\right)+2\left(x-x_{0}\right)\left(y-y_{0}\right) p_{1}+\left(r^{2}+2\left(y-y_{0}\right)^{2}\right) p_{2}
\end{array}\right.
$$

In the formulas, $\triangle \mathrm{x}$ and $\triangle \mathrm{y}$ are correction values of
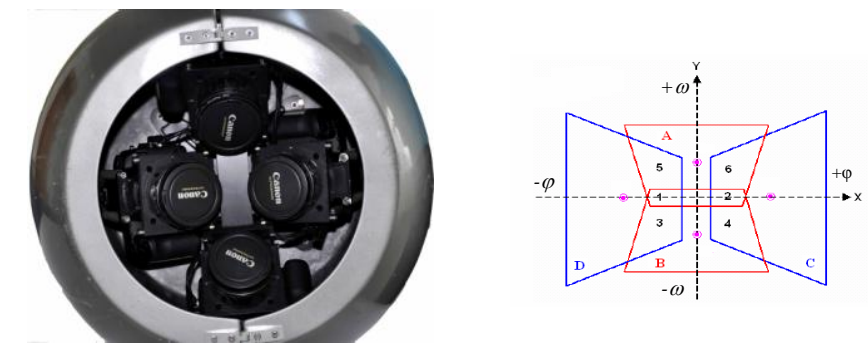

Fig.2. The combined camera LAC-04 and its combination mode

As shown in Figure 2, four cameras of A, B, C and D are inclined in pairs in opposite direction, and each camera is inclined in $\omega$ or $\varphi$ direction. A and B are only inclined with equal $\omega$ angle; $C$ and $D$ are only inclined with equal $\varphi$ angle. The purpose of this kind of geometric structure design is to realize self-calibration.

\section{B. Calibration of combined camera}

The purpose of combined camera is to obtain a wide virtual equivalent central projection image, which requires to accurately measure the values of relative orientation angles between combined cameras at the moment of exposure, so as to eliminate and restrict various distortions and errors of combined image. To eliminate the lens distortion of individual cameras and the deformation of mechanical structure, it is necessary to measure the relative location and orientation of each camera and to process the images through the process of combined camera calibration, which includes single camera calibration and combined calibration.

(1) Single camera calibration. It includes the measurement of the camera focal length (f), image principal point coordinate $\left(\mathrm{x}_{0}, \mathrm{y}_{0}\right)$ and various optical distortion coefficients. It uses the mathematical model of space resection, and this model is based on the collinearity equation and takes coordinates of image points as the observation values to calculate the exterior orientation elements, and interior orientation elements of camera, and lens distortion coefficients and other additional parameters. In image plane coordinate system, the camera image distortion correction formulas are as follow: 
point; $\left(\mathrm{x}_{0}, \mathrm{y}_{0}\right)$ is the image principal point coordinate; $r=\sqrt{\left(x-x_{0}\right)^{2}+\left(y-y_{0}\right)^{2}} ; \mathrm{k}_{1}, \mathrm{k}_{2}$, and $\mathrm{k}_{3}$ are radial distortion coefficients; $\mathrm{p}_{1}$ and $\mathrm{p}_{2}$ are tangential distortion coefficients; $b_{1}$ is non-square scale factor of pixel; $b_{2}$ is distortion coefficient of CCD array nonorthogonality.

(2) Combined calibration. Due to some errors and distortions caused by machine structure, it is necessary to measure the value of $\varphi 、 \omega 、 \kappa$ angles of four cameras by using combined calibration method, which includes initial calibration and self-calibration. The purpose of initial calibration is to identify the relative orientation angles of each single camera in the combined system. Four cameras are installed on a mechanical frame, and then take photo on calibration field. Exterior orientation element values of each camera are calculated by using space resection method, and then the relative orientation angle of each camera in the combined system could be calculated. This process is called initial calibration. The exterior orientation element values are used to transform the initial image to level image by reprojection.

Self-calibration. When deformation of mechanical components of combined camera occurs due to the change of temperature and platform movement, the values of relative orientation angles between four cameras calculated in initial calibration procedure may be changed. The change of relative spatial position of each camera (space shift) is usually small (sub-millimeter level), it causes small image distortion which could be ignored. However, the changes of relative orientation angle $(\varphi, \omega, \kappa)$ among four cameras may lead to large image distortion, which could not be ignored and need to be further calibrated. This calibration process is called self-calibration. The principle of self-calibration is that, after four images of A, $\mathrm{B}, \mathrm{C}$, and D are reprojected to the virtual level image, in the overlapping areas, the coordinates of the homonymy points of four images should be equal, using this condition to calculate the relative orientation angles of four cameras. The formula is listed as follows:

$$
\left\{\begin{array}{l}
\Delta X_{i j}=\frac{f^{2}+x_{i}^{2}}{f} \Delta \varphi_{i}+\frac{x_{i} y_{i}}{f} \Delta \omega_{i}-y_{i} \Delta \kappa_{i}-\frac{f^{2}+x_{j}^{2}}{f} \Delta \varphi_{j}-\frac{x_{j} y_{j}}{f} \Delta \omega_{j}+y_{j} \Delta \kappa_{j} \\
\Delta Y_{i j}=\frac{x_{i} y_{i}}{f} \Delta \varphi_{i}+\frac{f^{2}+y_{i}^{2}}{f} \Delta \omega_{i}+x_{i} \Delta \kappa_{i}-\frac{x_{j} y_{j}}{f} \Delta \varphi_{j}-\frac{f^{2}+y_{j}^{2}}{f} \Delta \omega_{j}-x_{j} \Delta \kappa_{j}
\end{array}\right.
$$

In the formula, $\mathrm{i}$ and $\mathrm{j}$ are the number of two cameras(e.g. A and C) that are adjacent and overlapped. While $\left(\mathrm{x}_{\mathrm{i}}, \mathrm{y}_{\mathrm{i}}\right),\left(\mathrm{x}_{\mathrm{j}}, \mathrm{y}_{\mathrm{j}}\right)$ are the image coordinates of a homonymy point in two camera images, $\left(\triangle X_{i j}, \triangle Y_{i j}\right)$ is the parallax of the homonymy point. Considerating the correlation of the four cameras in the unified coordinate system, there are following condition equations:

$$
\left\{\begin{array}{l}
\Delta \varphi_{A}+\Delta \varphi_{B}+\Delta \varphi_{C}+\Delta \varphi_{D}=0 \\
\Delta \omega_{A}+\Delta \omega_{B}+\Delta \omega_{C}+\Delta \omega_{D}=0 \\
\Delta \kappa_{A}+\Delta \kappa_{B}+\Delta \kappa_{C}+\Delta \kappa_{D}=0
\end{array}\right.
$$

The value of $\Delta \varphi, \Delta \omega$, and $\Delta \kappa$ can be acquired by solving the simultaneous equations of (2) and (3), then the relative orientation angle value could be corrected. To this step, the self-calibration could be achieved. Using the corrected value of $(\varphi, \omega, \kappa)$ to reproject the four images into level images, four images form a virtual equivalent central projection combined image without distortion.This process detects and calculates the accurate values of relative orientation angle of four cameras, that can be used to eliminates the distortions of the combined image caused by the temperature change and the platform movement, so the wide equivalent central projection combined image could be used in the following photogrammetric mapping.

\section{EXPERIMENT}

\section{A. Project overview}

The experiment site is along both sides of the Xiaoqinghe river in Jinan city, the area range is $6 \mathrm{~km}$ long and 500 meters wide. Aerial photography was performed by using the wide angle combined camera carried on the unmanned airship, the flight height is $200 \mathrm{~m}$. In this experiment, 5352 images were obtained, and 1338 wide combined images were generated, the image resolution (GSD) is $3.5 \mathrm{~cm}$.

\section{B. Results}

With the high resolution and wide images, the aerial triangulation was performed by MAP-AT software, 1:500 scale topographical map, DEM and DOM were produced on 
digital photogrammetry work station JX-4 and VirtuoZo. The planimetric coordinates and elevations of the object points are all meansured by office work rather than the elevation is measured by field work. The mapping accuracy is that: the planimetric RMSE(root mean square error) is $M x y= \pm 0.17 \mathrm{~m}$, the elevation $\mathrm{RMSE}$ is $\mathrm{Mz}= \pm 0.16 \mathrm{~m}$, and the distance RMSE of the adjacent ground points is $\mathrm{Md}= \pm 0.12 \mathrm{~m}$, the planimetric RMSE of DOM is $0.09 \mathrm{~m}$. The result meets the accuracy of 1:500 scale topographic mapping required by national standard. The DOM is showed in figure 3.
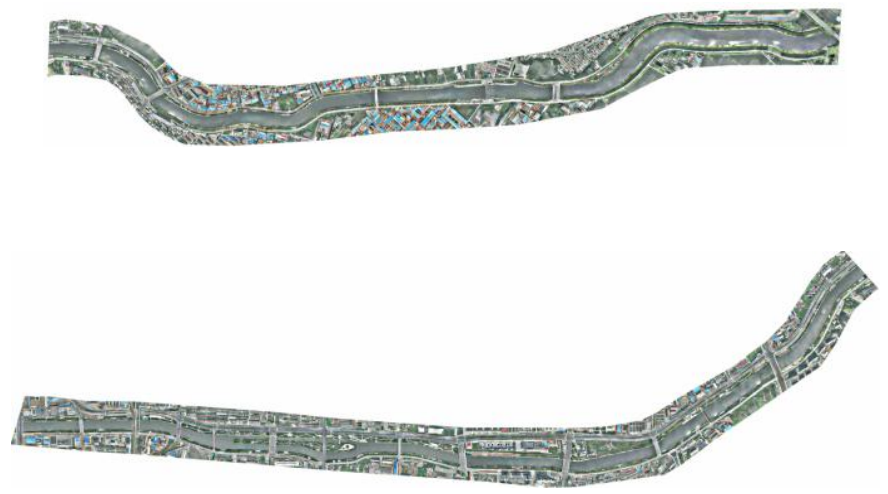

Fig. 3. DOM (above: the northeast segment; below: the west segment)

\section{CONCLUSION}

By using low altitude unmanned airship integrating the combined wide angle camera, high resolution and wide images are obtained, which improves the base-height ratio of stereo images, consequently, increases the accuracy of stereo mapping, espceially the elevation accuracy. The technology adopted in the experiment solved the existing problem of large scale mapping by photogrammetric method, that is, the elevation accuracy is difficult to meet the required accuracy in large scale topographic mapping by photogrammetric method, and this method greatly improve the production efficiency. The experiment indicates that, the low altitude unmanned airship photogrammetry is a good new remote sensing technology to obtain the high precision geographic information, and it is a practical and reliable method.

\section{REFERENCES}

[1] Fang Yong, Cui Weiping, Ma xiaofeng, Hu Haiyan. "Image Stitching Algorithm of a Digital Camera with Single Field
Lens and Multiple Area CCD," Geomatics and Information Science of Wuhan University, 2012, Vol. 37, No.8, pp. 907-910.

[2] Wang Hui, Wu Yusheng, Zhang Yongsheng, "Modeling and Analyzing of Geometric Joint Error for CCD Matrix Images of Digital Aerial Camera," Journal of Institute of Surveying and Mapping, 2003, Vol. 20,No.4. pp.257-262.

[3] Lin Zongjin. "UAV borne low altitude photogrammetry system," Science of Surveying and Mapping, 2011, Vol. 36, No.1, pp. 5-9.

[4] Zhang Zuxun. "Aspects on Aerial Digital cameras," Engineering of Surveying and Mapping, 2004, Vol. 13,No.4,pp. $1-5$.

[5] Lin Zongjin, Su Guozhong, Zhi Xiaodong. "UAV Borne Double Camera Low Altitude Photogrammetry System," Geospatial Information, 2010, Vol. 8,No.4, pp. 1-3.24.

[6] Li Jian, Liu Xian-lin, Liu Feng-de, et al. "Mosaic model of SWDC-4 large format aerial digital camera and accuracy analysis of stereo mapping," Science of Surveying and Mapping. 2008, Vol. 33,No.2, pp. 104-106.120.

[7] Peng Xiao-dong. "Research of Low Altitude Photogrammetry of Unmanned Airship," PH.D. Dissertation, Wuhan University, 2010.

[8] Liu Zhaoqin. "Study on wide-angle and light small combined digital camera system for UAV," Ph.D. Dissertation. Shandong University of Science and Technology, 2008.

[9] Jinjun Rao,Zhenbang Gong,Jun Luo and Shaorong Xie. "Unmanned Airships for Emergency Management," Proceedings of the 2005 IEEE International Workshop on Safety, Security and Rescue Robotics. Kobe,Japan. 2005, pp. 125-130.

[10] Frederic Guichard, Edwin Bourget, Jean-Paul Agnard. "High-Resolution Remote Sensing of International Ecosystems: A Low-Cost Technique to Link Scale-Dependent Patterns and Processes," Limnology and Oceanography. 2000, Vol. 45,No.2,pp.328-338 